\title{
Erratum: Ultrastrong coupling in the near field of complementary split-ring resonators [Phys. Rev. B 90, 205309 (2014)]
}

Curdin Maissen, Giacomo Scalari, Federico Valmorra, Sara Cibella, Roberto Leoni, Christian Reichl, Christophe Charpentier, Werner Wegscheider, Mattias Beck, and Jérôme Faist

(Received 14 August 2018; published 4 September 2018)

DOI: 10.1103/PhysRevB.98.119901

The equations reported in Sec. II C in the original paper contain multiple errors. First, the relative material permittivity $\epsilon$ has been accounted for twice: once directly in the definition of the electromagnetic vector potential [Eq. (2) in the original paper] and a second time via the definition of the effective volume [Eq. (3)]. Since $\epsilon$ is varying for the present case, it is convenient to account for its contribution via the effective volume. In this convention, the electromagnetic vector potential is

$$
\hat{\mathbf{A}}_{\mathrm{LC}}(\mathbf{r}, t)=\sqrt{\frac{\hbar}{2 \epsilon_{0} \omega V}}\left[\hat{a} \mathbf{u}(\mathbf{r}) e^{-i \omega t}+\hat{a}^{\dagger} \mathbf{u}^{*}(\mathbf{r}) e^{i \omega t}\right] .
$$

Second, in Eq. (4), the weight factor $\left(u_{x}+i u_{y}\right) / \sqrt{2}$ was omitted in the sum, and a factor of $\sqrt{2}$, accounting for the circular polarization selection rule of the cyclotron transition, was omitted in the normalization factor. The correct expression for the bright mode creation operator is

$$
\hat{b}^{\dagger}=\frac{1}{\sqrt{N_{e}}} \sum_{k} \frac{u_{x}+i u_{y}}{\sqrt{2}} \hat{c}_{v, k}^{\dagger} \hat{c}_{\nu-1, k}
$$

with

$$
N_{e}=\frac{\rho_{2 \mathrm{DEG}}}{v} \int_{2 \mathrm{DEG}} \frac{\left|u_{x}(\mathbf{r})\right|^{2}+\left|u_{y}(\mathbf{r})\right|^{2}}{2} d x d y .
$$

These two errors lead to a factor of $\sqrt{\epsilon v}$ in Eq. (6) which becomes

$$
\Omega_{R}=\sqrt{\frac{\omega_{c} e^{2} N_{e} v}{4 \epsilon_{0} \omega_{\mathrm{LC}} V m^{*}}} .
$$

Moreover, in Eq. (7) a factor of $\sqrt{\epsilon / 8 \pi}$ is missing

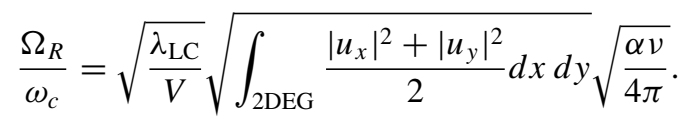

Values for the mode volume and effective number of coupled electrons calculated with the corrected formulas are $V=$ $1.3 \times 10^{-15} \mathrm{~m}^{3}$ and $N_{e}=5200$. For this calculation, the electric-field distribution $\mathbf{u}$ (calculated with the eigenfrequency solver of the COMSOL $\mathrm{rf}$ module) was normalized according to $\max _{2 \mathrm{DEG}}\left(\frac{\left|u_{x}+i u_{y}\right|^{2}}{2}\right)=1$. The resulting coupling rate of $\left(\Omega_{R} / \omega_{c}\right)_{B_{\mathrm{FEM}}}=0.22$ is independent of the chosen normalization condition, whereas both $V$ and $N_{e}$ are inversely proportional to the normalization value.

The remainder of the paper discusses relative changes in the normalized coupling rate $\frac{\Omega_{R}}{\omega_{c}}$. The erroneous factors cancel out for these quantities. Thus, the results and conclusions reported in the paper remain valid. 